\title{
Commentary
}

\section{Using the public health model to address unintentional injuries and TBI: A perspective from the Centers for Disease Control and Prevention (CDC)}

\author{
Grant Baldwin, Matt Breiding* and David Sleet \\ Division of Unintentional Injury Prevention, National Center for Injury Prevention and Control, \\ Centers for Disease Control and Prevention, Atlanta, GA, USA
}

\begin{abstract}
Traumatic brain injury (TBI) can have long term effects on mental and physical health, and can disrupt vocational, educational, and social functioning. TBIs can range from mild to severe and their effects can last many years after the initial injury. CDC seeks to reduce the burden of TBI from unintentional injuries through a focus on primary prevention, improved recognition and management, and intervening to improve health outcomes after TBI. CDC uses a 4-stage public health model to guide TBI prevention, moving from 1) surveillance of TBI, 2) identification of risk and protective factors for TBI, 3) development and testing of evidence-based interventions, to 4) bringing effective intervention to scale through widespread adoption. CDC's unintentional injury prevention activities focus on the prevention of sports-related concussions, motor vehicle crashes, and older adult falls. For concussion prevention, CDC developed Heads Up - an awareness initiative focusing on ways to prevent a concussion in sports, and identifying how to recognize and manage potential concussions. In motor vehicle injury prevention, CDC has developed a tool (MV PICCS) to calculate the expected number of injuries prevented and lives saved using various evidence-based motor vehicle crash prevention strategies. To help prevent TBI related to older adult falls, CDC has developed STEADI, an initiative to help primary care providers identify their patients' falls risk and provide effective interventions. In the future, $\mathrm{CDC}$ is focused on advancing our understanding of the public health burden of TBI through improved surveillance in order to produce more comprehensive estimates of the public health burden of TBI.
\end{abstract}

Keywords: Traumatic brain injury, unintentional injury, injury causes, injury prevention

\section{Introduction}

Unintentional injury can negatively impact anyone, regardless of age, race or economic status. More Americans die from unintentional injuries and vio-

*Address for correspondence: Matt Breiding, PhD, CDR, U.S. Public Health Service, Traumatic Brain Injury Team Lead, Division of Unintentional Injury Prevention, National Center for Injury Prevention \& Control, Centers for Disease Control \& Prevention, 4700 Buford Highway, MS F62, Atlanta, GA 30341, USA. Tel.: +1 770488 1396; E-mail: dvi8@cdc.gov. lence in the first three decades of life than from any other cause (Haegerich et al., 2014). Among all ages, unintentional injuries are the fourth leading cause of death with over 136,000 lives lost annually (Centers for Disease Control and Prevention, 2005). Millions of others suffer a non-fatal injury each year; due to an unintentional injury more than 3 million people are hospitalized and more than 27 million people are treated in emergency departments and released each year (Centers for Disease Control and Prevention, 2015a). 
A traumatic brain injury (TBI) can be one of the most debilitating injuries because of the potential for negative long-term effects on a person's mental and physical status, including their vocational, motor, behavioral and social/interpersonal functioning (Hoofien et al., 2001). In 2010, there were at least 2.5 million emergency department visits, hospitalizations, or deaths related to a TBI in the U.S., either alone or in combination with other injuries. Every hour, there are an average of 204 TBI-related emergency department visits, 33 TBI-related hospitalizations; and 6 TBI-related deaths (Centers for Disease Control and Prevention, 2015b). TBIs can occur in a variety of ways, ranging from a car crash, a fall in the bathtub, a collision with another player in a soccer game, an assault or suicide attempt, or from military engagement on the battle field. Falls and being struck by or against an object are the leading causes of TBI (Centers for Disease Control and Prevention, 2015b).

The severity of a TBI may range from mild to severe. The greater the severity of injury, the greater the likelihood of long-term and life-changing disability or even death. Most people who suffer a concussion, or mild TBI, recover within one or two weeks. For some however, symptoms can last for several weeks, months, or longer as the severity of the injury increases. TBI-related symptoms can affect all aspects of a person's life - at home, at school, or at work. They can lead to short- or long-term problems with how someone acts, feels, and thinks that can impact memory, behavior, mood, family dynamics, activities of daily living, and sleep patterns. TBIs, however, are largely preventable.

There is growing recognition that experiencing a TBI is not a single injury event, but can manifest in similar ways as a "chronic condition," having lasting effects many years after the initial injury (Masel \& DeWitt, 2010). While we are learning more each day about the long term health outcomes and impacts from a TBI, there is much that we still do not know. Rehabilitation experts are helping us to better understand how to promote healthy lifestyles among individuals living with the effects of a TBI, often through finding ways to reduce barriers for community participation.

For more than 20 years, the CDC Injury Center has been a leader in reducing the burden of injuries across the lifespan. As the nation's public health protection agency, CDC works to save lives, protect people, and save health care costs through prevention. CDC uses the four-stage public health model to guide our work across the agency. In this model, we move from public health surveillance to the identification of risk and protective factors to the development and implementation of effective programs and policies. Finally, we work to bring proven, evidence-based interventions to scale. Regardless of the topic - from infectious disease to chronic disease and injury prevention - the same framework guides our work.

Beginning in 1996 when Congress passed the TBI Act (Public Law 104-66), CDC was directed to help reduce the burden of death and disability caused by TBIs. The TBI Act, which has been amended and renewed three times since then, specifies certain activities as CDC's focus. Currently, CDC's work is focused in four areas including: (1) improving the understanding of the public health burden of TBI; (2) reducing the incidence of TBI through primary prevention; (3) improving the recognition and management of mild TBI; and (4) promoting healthy lifestyles and improving health outcomes of persons living with TBI. CDC's current work in TBI prevention includes activities in the prevention of sports and recreation-related concussion, motor vehicle injury prevention, and older adult fall prevention.

\section{Sports and recreation-related concussion prevention}

Sports and recreation-related concussions are serious injuries, with an estimated 325,000 children and teens treated in emergency departments for sports and recreation-related TBIs in 2012 (Coronado et al., 2015). That's just shy of 40 injuries every hour. Part of the CDC response is to develop, implement, and evaluate the effectiveness of large-scale efforts to increase knowledge about concussions and change the culture surrounding concussions.

Ten years ago, CDC introduced the Heads $U p$ initiative. It was developed to better equip young athletes, parents, health care providers, coaches, and teachers to prevent, recognize, and respond to concussions. Heads $U p$ is focused on how to prevent a brain injury, and what to do if there is concern as to whether someone has suffered a concussion during play. The goal of Heads $U p$ is to protect children and adolescents from concussions by raising awareness, enhancing knowledge, and informing action to improve prevention, recognition, and response to concussions. This is done by translating the latest concussion science into educational products tailored specifically for the target audiences, and working 
with partner organizations to disseminate and integrate concussion education materials and messages into their existing systems and programs. The reach this program has achieved is staggering. $\mathrm{CDC}$ has created over 50 products, distributed more than 6 million copies of our Heads Up materials, and trained over 3 million coaches. The entire suite of Heads Up materials can be found, free of charge, at http://www.cdc.gov/headsup.

\section{Motor vehicle injury prevention}

After the age of one, motor vehicle injury is either the first or second leading cause of injury death among all age groups across the lifespan (Centers for Disease Control and Prevention, 2005) and a leading cause of TBI. For youth and young adults motor vehicles are the main cause of TBI-related death, accounting for $55.8 \%$ of TBI deaths in those ages 5 to 14 years old and $47.4 \%$ of TBI-related deaths for those ages 15 to 24 years old (Centers for Disease Control and Prevention, 2015c). In 2014 nearly 33,000 Americans died in a motor vehicle-related crash (National Highway Traffic Safety Administration, 2014). It is the fifth leading cause of years of potential life lost (YPLL) and accounts for 10 percent of all YPLL before the age of 65 when all causes of death are included (Centers for Disease Control and Prevention, 2005). At CDC, we aim to keep people safe on the roads everyday - drivers, passengers, and pedestrians. Our role includes research on what works to prevent motor vehicle crashes and the promotion of proven programs and policies that protect people from injury and death. We accomplish this through a multifaceted program of applied research, implementation, evaluation, and translation of effective interventions. Our work complements the work of the National Highway Traffic Safety Administration (NHTSA) and other federal partners in the transportation safety community.

One current project is a tool for state decisionmakers. CDC recently released a new, easy-to-use, interactive, online calculator, called the Motor Vehicle PICCS (Prioritizing Interventions and Cost Calculator for States) to help legislators and other state officials prioritize and select from a suite of 14 effective motor vehicle injury prevention interventions - including primary enforcement seat belt laws, bicycle helmet laws, and universal motorcycle helmet laws. MV PICCS is designed to calculate the expected number of injuries prevented and lives saved at the state level, the monetized value of the number of injuries prevented and lives saved, and the costs of implementation. Preventing MV crashes and the injuries they inflict will help reduce the overall burden of TBI. To use MV PICCs go to http://www.cdc.gov/ motorvehiclesafety/calculator.

\section{Older adult fall prevention}

Falls are another common cause of a TBI. More than $80 \%$ of TBIs in adults over the age of 65 are caused by falls (Centers for Disease Control and Prevention, 2005). From 2006-2010, falls accounted for $40 \%$ of all TBIs in the U.S. that resulted in an ED visit, hospitalization, or death (Centers for Disease Control and Prevention, 2015d). In 2014 over 27,000 older adults died from falls (Centers for Disease Control and Prevention, 2005) and about half of these fatalities were due to TBIs. Falls also result in significant direct medical costs and are among the 20 most costly medical conditions (Carroll, Slattum, \& Cox, 2005); approximately $\$ 34$ billion dollars a year are spent on direct medical costs alone (Stevens, Corso, Finkelstein \& Miller, 2006).

Falls resulting in a TBI not only affect a person's overall health but also their quality of life. Seniors may restrict their activities because of a fear of falling or as a result of a fall that resulted in a TBI. In turn, this fear of another fall can lead to decreased physical activity, reduced mobility, muscle weakness, fewer social interactions with friends and family, and decreased physical activity. A fear of falling can lead to a downward spiral resulting in the worsening of an older adult's overall health (Scheffer et al., 2008).

One out of every three older adults fall each year (Morrison et al., 2013), but less than half of those who fall talk to their healthcare providers about it (Stevens et al., 2012). Health care and primary care providers can play an important role in identifying important modifiable risk factors for falls and providing guidance about how to prevent falls and TBIs as a result of falls. For instance, the health care setting can be a place where patients at risk of falling are identified and then referred to appropriate evidence-based community fall prevention programs.

CDC also recognized the importance of fall prevention screening, assessment, treatment, and referral as a routine part of clinical care. We know that clinical assessment by a health care provider, combined with individualized treatment of identified risk factors, reduces the rate of falls by almost $25 \%$ (CDC 
Compendium, 2016). CDC's work can help simplify, harmonize, standardize, and automate systems, as well as provide evidence-based practice protocols for improved screening, preventive services, treatment, and outcomes. If we can train and provide physicians with effective tools, they can be a significant, trusted, and front-line resource in reducing falls and TBI.

One outgrowth of CDC's fall prevention efforts is the STEADI initiative (Stopping Elderly Accidents Deaths and Injuries). STEADI is focused on primary care providers who see older adults in their practice and uses established clinical guidelines and scientifically-tested interventions to help providers address their patients' fall risk, identify modifiable risk factors, and provide effective interventions. This includes interventions such as medication management and Vitamin D supplementation. STEADI is an integrated and team-driven approach and the potential impact is substantial. CDC estimated that for every 5,000 health care providers who adopt STEADI, over a 5 -year period as many as 6 million more patients could be screened, 1 million more falls could be prevented, and $\$ 3.5$ billion direct medical costs could be saved (Centers for Disease Control and Prevention, 2016). By linking the medical community with the public health and aging services communities, we can prevent more falls and ensure our nation's seniors remain active and live independently as long as possible. More information about STEADI can be found at http://www.cdc.gov/steadi/.

\section{Conclusion}

$\mathrm{CDC}$ is poised to reduce the incidence and burden of TBI by using the public health model to reduce sports-related concussion, motor vehicle injuries, and older adult falls and to improve health outcomes for individuals who are living with TBI. In addition to implementing programs - like those described above - CDC is developing approaches that will advance a national system to improve surveillance of TBI across the lifespan and from all causes. The system envisioned will capture TBIs that are missed by current healthcare-based data sources and provide national incidence estimates of TBI. Just as important, the surveillance system has been designed to provide true national estimates of TBI-related disability for the first time. Improved surveillance will give better estimates of the public health burden of TBI, and trend data will allow us to track whether the burden of TBI is increasing or decreasing, pro- viding evidence as to the effectiveness of prevention efforts.

There is much work left to be done in primary and secondary prevention of TBI caused by an unintentional injury, in acute care and rehabilitation, and in improving TBI surveillance and data collection. The CDC Injury Center will be working in the years to come to reduce the burden and consequences of TBI on individuals, families, and communities.

\section{Conflict of interest}

The authors have no conflict of interest to report.

Disclaimer: The findings and conclusions in this report are those of the authors and do not necessarily represent the views of the Centers for Disease Control and Prevention.

\section{References}

Carroll, N. V., Slattum, P. W., \& Cox, F. M. (2005). The cost of falls among the community-dwelling elderly. J Managed Care Pharmacy, 11, 307-316.

CDC Compendium of Effective Fall Interventions: What Works for Community-Dwelling Older Adults, 3rd Edition. Available at: http://www.cdc.gov/homeandrecreationalsafety/ falls/compendium.html. Accessed 3/14/2016.

Centers for Disease Control and Prevention, National Center for Injury Prevention and Control (2005). Web-based Injury Statistics Query and Reporting System (WISQARS) [online]. (2005) [cited 2016 Mar 8]. Available from URL: www.cdc.gov/ injury/wisqars

Centers for Disease Control and Prevention (2015a), About CDC's Injury center, accessed on 12-22-15 at http://www.cdc.gov/ injury/about/index.html.

Centers for Disease Control and Prevention (2015b). Traumatic Brain Injury in the United States: Fact Sheet [Internet].CDC.gov.Atlanta (GA): CDC; [updated 2015 Jan 12]. Available from: http://www.cdc.gov/traumaticbraininjury/get_ the_facts.html.

Centers for Disease Control and Prevention (2015c). TBI Data and Statistics. Available at: http://www.cdc.gov/ traumaticbraininjury/data/index.html. Accessed April, 2015.

Centers for Disease Control and Prevention (2015d). Traumatic Brain Injury in the United States: Fact Sheet [Internet].CDC.gov. Atlanta (GA): CDC; [updated 2015 Jan 12]. Available from http://www.cdc.gov/traumaticbraininjury/ data/dist_ed.html.

Centers for Disease Control and Prevention (2016). Unpublished estimates available at: http://www.cdc.gov/steadi/. Accessed 3/23/2016.

Coronado, V.G., Haileyesus, T., Cheng, T.A., Bell, J.M., Haarbauer-Krupa, J., Lionbarger, M.R., Flores-Herrera, J., McGuire, L.C. and Gilchrist, J. (2015). Trends in sports-and recreation-related traumatic brain injuries treated in US emer- 
gency departments: the National Electronic Injury Surveillance System-All Injury Program (NEISS-AIP) 2001-2012. The Journal of Head Trauma Rehabilitation, 30(3), 185-197.

Haegerich, T. M., Dahlberg, L. L., Simon, T. R., Baldwin, G. T., Sleet, D. A., Greenspan, A. L., \& Degutis, L. C. (2014). Prevention of injury and violence in the USA. The Lancet, 9937, 64-74.

Hoofien, D., Gilboa, A., Vakil, E., \& Donovick, P. J. (2001). Traumatic brain injury (TBI) 10-20 years later: A comprehensive outcome study of psychiatric symptomatology, cognitive abilities and psychosocial functioning. Brain Injury, 15(3), 189-209. DOI 10.1080/026990501300005659

Masel, B. E., \& DeWitt, D. S. (2010). TBI: A disease process not an event. Journal of Neurotrauma, 27(8), 1529-1540.

Morrison, A., Fan, T., Sen, S. S., \& Weisenfluh L. (2013). Epidemiology of falls and osteoporotic fractures: A systematic review. Clinicoecon Outcomes Res, 5, 9-18.
National Highway Traffic Safety Administration. Quick Facts 2014. Available from URL: http://www-nrd.nhtsa.dot.gov/ Pubs/812234.pdf

Scheffer, A. C., Schuurmans, M. J., Van Dijk, N., Van Der Hooft, T., \& De Rooij, S. E. (2008). Fear of falling: Measurement strategy, prevalence, risk factors and consequences among older persons. Age Ageing, 37(1), 19-24.

Stevens, J. A., Ballesteros, M. F., Mack, K. A., Rudd, R. A., DeCaro, E., \& Adler, G. (2012). Gender differences in seeking care for falls in the aged Medicare population. American Journal of Preventive Medicine, 43(1), 59-62.

Stevens, J. A., Corso, P. S., Finkelstein, E. A., \& Miller, T. R. (2006). The costs of fatal and nonfatal falls among older adults. Injury Prevention, 12, 290-295. 